\title{
Lyapunov-type inequalities for higher-order half-linear difference equations
}

Haidong Liu ${ }^{1 *}$

\section{"Correspondence:}

tomlhd983@163.com

'School of Mathematical Sciences,

Qufu Normal University, Qufu, China

\begin{abstract}
In this paper, we will establish some new Lyapunov-type inequalities for some higher-order superlinear-sublinear difference equations with boundary conditions. Our results not only complement the existing results established in the literature, but also furnish a handy tool for the study of qualitative properties of solutions of some difference equations.
\end{abstract}

Keywords: Lyapunov-type inequality; Difference equation; Higher-order; Boundary conditions

\section{Introduction}

In recent years, there has been an increasing interest in obtaining various classes of inequalities, which play an important role in qualitative analysis of solutions to differential and difference equations; see [1-27]. In the field of inequalities, the Lyapunov-type inequality is one of the fundamental inequalities, which was initially investigated by Lyapunov in 1907. After having been discovered, the Lyapunov inequality and its various generalizations were extensively studied by numerous mathematicians. This is due to the fact that these inequalities have proved to be useful tools in the study of oscillation theory, disconjugacy, eigenvalue problems, and many directions of mathematics research areas. For some recent work, the reader is referred to [28-45] and the references therein. In particular, Liu and Tang [37] studied the following $m$-order $p$-Laplace difference equation:

$$
\left|\Delta^{m} u(n)\right|^{p-2} \Delta^{m} u(n)+r(n)|u(n)|^{p-2} u(n)=0,
$$

where $m \in \mathbb{N}, n \in \mathbb{Z}$ and $r(n)$ is a real-valued function defined on $\mathbb{Z}, p>1$ is a constant, $\Delta$ denotes the forward difference operator defined by $\Delta x(n)=x(n+1)-x(n)$, and $u(n)$ satisfies the following anti-periodic boundary conditions:

$$
\Delta^{i} u(a)+\Delta^{i} u(b)=0, \quad i=0,1, \ldots, m-1 ; \quad u(n) \not \equiv 0, \quad n \in \mathbb{Z}[a, b] .
$$

(c) The Author(s) 2020. This article is licensed under a Creative Commons Attribution 4.0 International License, which permits use, sharing, adaptation, distribution and reproduction in any medium or format, as long as you give appropriate credit to the original author(s) and the source, provide a link to the Creative Commons licence, and indicate if changes were made. The images or other third party material in this article are included in the article's Creative Commons licence, unless indicated otherwise in a credit line to the material. If material is not included in the article's Creative Commons licence and your intended use is not permitted by statutory regulation or exceeds the permitted use, you will need to obtain permission directly from the copyright holder. To view a copy of this licence, visit http://creativecommons.org/licenses/by/4.0/. 
Recently, Liu [43] established a new discrete Lyapunov-type inequality for the following generalized $m$-order $p$-Laplace difference equation with mixed non-linearities:

$$
\left|\Delta^{m} u(n)\right|^{p-2} \Delta^{m} u(n)+\sum_{i=0}^{m-1} r_{i}(n)\left|\Delta^{i} u(n)\right|^{p-2} \Delta^{i} u(n)=0
$$

with the anti-periodic boundary conditions (2), where $m \in \mathbb{N}, n \in \mathbb{Z}, p>1$ is a constant and $r_{i}(n)(i=0,1, \ldots, m-1)$ are real-valued functions defined on $\mathbb{Z}$.

However, to the best of our knowledge, Lyapunov-type inequalities for the superlinearsublinear difference equation have received less attention. The main goal of this paper is to use the Hölder inequality and other inequalities to establish Lyapunov-type inequalities for superlinear-sublinear difference equation of the form

$$
\begin{aligned}
& \left|\Delta^{m} u(n)\right|^{\alpha-2} \Delta^{m} u(n)+q(n)\left|\Delta^{m} u(n)\right|^{\beta-2} \Delta^{m} u(n)-r(n)|u(n)|^{\gamma-2} u(n)=0, \\
& \quad n \in \mathbb{Z}[a, b],
\end{aligned}
$$

with the anti-periodic boundary conditions (2), and superlinear-sublinear difference equation of the form

$$
\begin{aligned}
& \left|\Delta^{2 m} u(n)\right|^{\alpha-2} \Delta^{2 m} u(n)+q(n)\left|\Delta^{2 m} u(n)\right|^{\beta-2} \Delta^{2 m} u(n)-r(n)|u(n)|^{\gamma-2} u(n)=0, \\
& \quad n \in \mathbb{Z}[a, b],
\end{aligned}
$$

with the following boundary conditions:

$$
\Delta^{2 i} u(a)=\Delta^{2 i} u(b)=0, \quad i=0,1, \ldots, m-1 ; \quad u(n) \not \equiv 0, \quad n \in \mathbb{Z}[a, b],
$$

where $m \in \mathbb{N}, 1<\alpha<\gamma<\beta$ are constants, $r(n)$ and $q(n)$ are real-valued functions defined on $\mathbb{Z}$ with $q(n)>0$.

Our results not only complement the existing results established in the literature, such as those in $[37,39,43]$, but also furnish a handy tool for the study of qualitative properties of solutions of some difference equations.

\section{Main results}

In what follows, we always assume that $a, b \in \mathbb{N}, \mathbb{Z}[a, b]=\{a, a+1, \ldots, b-1, b\}$ and $\mathbb{Z}(a, b)=$ $\{a+1, a+2, \ldots, b-2, b-1\}$.

Lemma 2.1 Let $M>0, N>0, \lambda>0$ and $\theta>0$ be given and $1<\lambda<\theta$. Then, for each $x \geq 0$,

$$
M x^{\lambda}-N x^{\theta} \leq \frac{M(\theta-\lambda)}{\theta-1}\left(\frac{(\theta-1) N}{(\lambda-1) M}\right)^{(\lambda-1) /(\lambda-\theta)} x
$$

holds.

Proof If $x=0$, then it is easy to see that the inequality (7) holds. So we only prove the inequality (7) holds in the case of $x>0$. Set $F(x)=M x^{\lambda-1}-N x^{\theta-1}, x>0$. Let $F^{\prime}(x)=0$, we 
get $x_{0}=\left(\frac{M(\lambda-1)}{N(\theta-1)}\right)^{1 /(\theta-\lambda)}$. Since $\forall x \in\left(0, x_{0}\right), F^{\prime}(x)>0 ; \forall x \in\left(x_{0},+\infty\right), F^{\prime}(x)<0, F$ obtains its maximum at $x_{0}=\left(\frac{M(\lambda-1)}{N(\theta-1)}\right)^{1 /(\theta-\lambda)}$ and $F_{\max }=F\left(x_{0}\right)=\frac{M(\theta-\lambda)}{\theta-1}\left(\frac{(\theta-1) N}{(\lambda-1) M}\right)^{(\lambda-1) /(\lambda-\theta)}$. Hence we get

$$
M x^{\lambda-1}-N x^{\theta-1} \leq \frac{M(\theta-\lambda)}{\theta-1}\left(\frac{(\theta-1) N}{(\lambda-1) M}\right)^{(\lambda-1) /(\lambda-\theta)}
$$

i.e.,

$$
M x^{\lambda}-N x^{\theta} \leq \frac{M(\theta-\lambda)}{\theta-1}\left(\frac{(\theta-1) N}{(\lambda-1) M}\right)^{(\lambda-1) /(\lambda-\theta)} x,
$$

then (7) holds. The proof is complete.

Lemma $2.2([39])$ Assume that $u(n)$ is a real-valued function on $\mathbb{Z}[a, b], u(a)=u(b)=0$. Then

$$
\begin{aligned}
& |u(n)| \leq \frac{(n-a)(b-n)}{b-a} \sum_{s=a}^{b-1}\left|\Delta^{2} u(s)\right|, \quad \forall n \in \mathbb{Z}[a, b-1], \\
& \sum_{n=a}^{b-1}|u(n)| \leq \frac{1}{2} \sum_{n=a}^{b-1}\left[(n-a+1)(b-n-1)\left|\Delta^{2} u(n)\right|\right] \leq \frac{(b-a)^{2}}{8} \sum_{n=a}^{b-1}\left|\Delta^{2} u(n)\right|, \\
& \forall n \in \mathbb{Z}[a, b-1] .
\end{aligned}
$$

Theorem 2.1 If $u(n)$ is a nonzero solution of Eq. (4) satisfying the anti-periodic boundary conditions (2), then

$$
1 \leq \Theta\left(\frac{\gamma-\alpha}{q_{0}(\beta-\alpha)}\right)^{(\gamma-\alpha) /(\beta-\gamma)} \frac{(\beta-\gamma)}{\beta-\alpha}\left(\sum_{n=a}^{b-1}|r(n)|^{\alpha /(\alpha-1)}\right)^{(\alpha-1)(\beta-\alpha) /[\alpha(\beta-\gamma)]},
$$

where

$$
\begin{aligned}
& q_{0}=\min _{n \in \mathbb{Z}[a, b]}\{q(n)\}, \\
& \Theta=(b-a)^{(\beta-\alpha)(m \alpha \gamma-m \alpha+1-\alpha) /[\alpha(\beta-\gamma)]} 2^{m(\gamma-1)(\alpha-\beta) /(\beta-\gamma)} .
\end{aligned}
$$

Proof Since the nonzero solution $u(n)$ of Eq. (4) satisfies the anti-periodic boundary conditions (2), then $u(a)+u(b)=0$. For $n \in \mathbb{Z}[a, b]$, we have

$$
\begin{aligned}
u(n) & =u(n)-\frac{1}{2}[u(a)+u(b)]=\frac{1}{2} \sum_{k=a}^{n-1}[u(k+1)-u(k)]-\frac{1}{2} \sum_{k=n}^{b-1}[u(k+1)-u(k)] \\
& =\frac{1}{2} \sum_{k=a}^{n-1} \Delta u(k)-\frac{1}{2} \sum_{k=n}^{b-1} \Delta u(k) .
\end{aligned}
$$

Then

$$
|u(n)| \leq \frac{1}{2} \sum_{k=a}^{b-1}|\Delta u(k)|
$$


Similarly, we get

$$
\left|\Delta^{i} u(n)\right| \leq \frac{1}{2} \sum_{k=a}^{b-1}\left|\Delta^{i+1} u(k)\right|, \quad i=1,2, \ldots, m-1 .
$$

Then, from (14) and (15), we have

$$
|u(n)| \leq\left(\frac{1}{2}\right)^{m}(b-a)^{m-1} \sum_{k=a}^{b-1}\left|\Delta^{m} u(k)\right|
$$

Multiplying (4) by $\Delta^{m} u(n)$, we obtain

$$
\left|\Delta^{m} u(n)\right|^{\alpha}=r(n)|u(n)|^{\gamma-2} u(n) \Delta^{m} u(n)-q(n)\left|\Delta^{m} u(n)\right|^{\beta}, \quad n \in \mathbb{Z}[a, b] .
$$

Then we get

$$
\begin{aligned}
\left|\Delta^{m} u(n)\right|^{\alpha} & =r(n)|u(n)|^{\gamma-2} u(n) \Delta^{m} u(n)-q(n)\left|\Delta^{m} u(n)\right|^{\beta} \\
& \leq|r(n)||u(n)|^{\gamma-1}\left|\Delta^{m} u(n)\right|-q(n)\left|\Delta^{m} u(n)\right|^{\beta} .
\end{aligned}
$$

Summing (18) from $a$ to $b-1$, we have

$$
\sum_{n=a}^{b-1}\left|\Delta^{m} u(n)\right|^{\alpha} \leq \sum_{n=a}^{b-1}|r(n)||u(n)|^{\gamma-1}\left|\Delta^{m} u(n)\right|-\sum_{n=a}^{b-1} q(n)\left|\Delta^{m} u(n)\right|^{\beta} .
$$

For the first summation on the right-hand side of (19), from (16) we obtain

$$
\begin{aligned}
& \sum_{n=a}^{b-1}|r(n)||u(n)|^{\gamma-1}\left|\Delta^{m} u(n)\right| \\
& \quad \leq\left(\frac{1}{2}\right)^{m(\gamma-1)}(b-a)^{(m-1)(\gamma-1)}\left(\sum_{n=a}^{b-1}\left|\Delta^{m} u(n)\right|\right)^{\gamma-1} \sum_{n=a}^{b-1}|r(n)|\left|\Delta^{m} u(n)\right| .
\end{aligned}
$$

On the other hand, from the discrete Hölder inequality,

$$
\sum_{n=a}^{b-1}|f(n) g(n)| \leq\left(\sum_{n=a}^{b-1}|f(n)|^{\rho}\right)^{1 / \rho}\left(\sum_{n=a}^{b-1}|g(n)|^{\nu}\right)^{1 / \nu}
$$

with $f(n)=1, g(n)=\left|\Delta^{m} u(n)\right|, \rho=\alpha /(\alpha-1)$ and $v=\alpha$, we have

$$
\begin{aligned}
\sum_{n=a}^{b-1}\left|\Delta^{m} u(n)\right| & \leq\left(\sum_{n=a}^{b-1} 1^{\alpha /(\alpha-1)}\right)^{(\alpha-1) / \alpha}\left(\sum_{n=a}^{b-1}\left|\Delta^{m} u(n)\right|^{\alpha}\right)^{1 / \alpha} \\
& =(b-a)^{(\alpha-1) / \alpha}\left(\sum_{n=a}^{b-1}\left|\Delta^{m} u(n)\right|^{\alpha}\right)^{1 / \alpha}
\end{aligned}
$$


and, with $f(n)=|r(n)|, g(n)=\left|\Delta^{m} u(n)\right|, \rho=\alpha /(\alpha-1)$ and $\nu=\alpha$, we get

$$
\sum_{n=a}^{b-1}|r(n)|\left|\Delta^{m} u(n)\right| \leq\left(\sum_{n=a}^{b-1}|r(n)|^{\alpha /(\alpha-1)}\right)^{(\alpha-1) / \alpha}\left(\sum_{n=a}^{b-1}\left|\Delta^{m} u(n)\right|^{\alpha}\right)^{1 / \alpha} .
$$

Then, from (20), (22) and (23), we obtain

$$
\begin{aligned}
& \sum_{n=a}^{b-1}|r(n)||u(n)|^{\gamma-1}\left|\Delta^{m} u(n)\right| \\
& \leq\left(\frac{1}{2}\right)^{m(\gamma-1)}(b-a)^{(m-1 / \alpha)(\gamma-1)}\left(\sum_{n=a}^{b-1}\left|\Delta^{m} u(n)\right|^{\alpha}\right)^{(\gamma-1) / \alpha} \\
& \quad \cdot\left(\sum_{n=a}^{b-1}|r(n)|^{\alpha /(\alpha-1)}\right)^{(\alpha-1) / \alpha}\left(\sum_{n=a}^{b-1}\left|\Delta^{m} u(n)\right|^{\alpha}\right)^{1 / \alpha} \\
& =\left(\frac{1}{2}\right)^{m(\gamma-1)}(b-a)^{(m-1 / \alpha)(\gamma-1)}\left(\sum_{n=a}^{b-1}|r(n)|^{\alpha /(\alpha-1)}\right)^{(\alpha-1) / \alpha}\left(\sum_{n=a}^{b-1}\left|\Delta^{m} u(n)\right|^{\alpha}\right)^{\gamma / \alpha} .
\end{aligned}
$$

Combining (11), (19) with (24), we get

$$
\begin{aligned}
& \sum_{n=a}^{b-1}\left|\Delta^{m} u(n)\right|^{\alpha} \\
& \leq\left(\frac{1}{2}\right)^{m(\gamma-1)}(b-a)^{(m-1 / \alpha)(\gamma-1)}\left(\sum_{n=a}^{b-1}|r(n)|^{\alpha /(\alpha-1)}\right)^{(\alpha-1) / \alpha}\left(\sum_{n=a}^{b-1}\left|\Delta^{m} u(n)\right|^{\alpha}\right)^{\gamma / \alpha} \\
& \quad-\sum_{n=a}^{b-1} q(n)\left|\Delta^{m} u(n)\right|^{\beta} \\
& \leq\left(\frac{1}{2}\right)^{m(\gamma-1)}(b-a)^{(m-1 / \alpha)(\gamma-1)}\left(\sum_{n=a}^{b-1}|r(n)|^{\alpha /(\alpha-1)}\right)^{(\alpha-1) / \alpha}\left(\sum_{n=a}^{b-1}\left|\Delta^{m} u(n)\right|^{\alpha}\right)^{\gamma / \alpha} \\
& \quad-q_{0} \sum_{n=a}^{b-1}\left|\Delta^{m} u(n)\right|^{\beta} .
\end{aligned}
$$

On the other hand, by Hölder inequality (21) with $f(n)=1, g(n)=\left|\Delta^{m} u(n)\right|^{\alpha}, \rho=\beta /(\beta-\alpha)$ and $v=\beta / \alpha$, we have

$$
\begin{aligned}
\sum_{n=a}^{b-1}\left|\Delta^{m} u(n)\right|^{\alpha} & \leq\left(\sum_{n=a}^{b-1} 1^{\beta /(\beta-\alpha)}\right)^{(\beta-\alpha) / \beta}\left(\sum_{n=a}^{b-1}\left(\left|\Delta^{m} u(n)\right|^{\alpha}\right)^{\beta / \alpha}\right)^{\alpha / \beta} \\
& =(b-a)^{(\beta-\alpha) / \beta}\left(\sum_{n=a}^{b-1}\left|\Delta^{m} u(n)\right|^{\beta}\right)^{\alpha / \beta} .
\end{aligned}
$$

Therefore,

$$
(b-a)^{(\alpha-\beta) / \alpha}\left(\sum_{n=a}^{b-1}\left|\Delta^{m} u(n)\right|^{\alpha}\right)^{\beta / \alpha} \leq \sum_{n=a}^{b-1}\left|\Delta^{m} u(n)\right|^{\beta} .
$$


From (25) and (27), we get

$$
\begin{aligned}
& \sum_{n=a}^{b-1}\left|\Delta^{m} u(n)\right|^{\alpha} \\
& \leq\left(\frac{1}{2}\right)^{m(\gamma-1)}(b-a)^{(m-1 / \alpha)(\gamma-1)}\left(\sum_{n=a}^{b-1}|r(n)|^{\alpha /(\alpha-1)}\right)^{(\alpha-1) / \alpha}\left(\sum_{n=a}^{b-1}\left|\Delta^{m} u(n)\right|^{\alpha}\right)^{\gamma / \alpha} \\
& \quad-(b-a)^{(\alpha-\beta) / \alpha} q_{0}\left(\sum_{n=a}^{b-1}\left|\Delta^{m} u(n)\right|^{\alpha}\right)^{\beta / \alpha} .
\end{aligned}
$$

For the right-hand of (28), from the inequality (7) in Lemma 2.1, with

$$
M=\left(\frac{1}{2}\right)^{m(\gamma-1)}(b-a)^{(m-1 / \alpha)(\gamma-1)}\left(\sum_{n=a}^{b-1}|r(n)|^{\alpha /(\alpha-1)}\right)^{(\alpha-1) / \alpha},
$$

$x=\sum_{n=a}^{b-1}\left|\Delta^{m} u(n)\right|^{\alpha}, N=(b-a)^{(\alpha-\beta) / \alpha} q_{0}, \lambda=\frac{\gamma}{\alpha}$, and $\theta=\frac{\beta}{\alpha}$, we get

$$
\begin{aligned}
& \left(\frac{1}{2}\right)^{m(\gamma-1)}(b-a)^{(m-1 / \alpha)(\gamma-1)}\left(\sum_{n=a}^{b-1}|r(n)|^{\frac{\alpha}{\alpha-1}}\right)^{(\alpha-1) / \alpha}\left(\sum_{n=a}^{b-1}\left|\Delta^{m} u(n)\right|^{\alpha}\right)^{\gamma / \alpha} \\
& \quad-(b-a)^{(\alpha-\beta) / \alpha} q_{0}\left(\sum_{n=a}^{b-1}\left|\Delta^{m} u(n)\right|^{\alpha}\right)^{\beta / \alpha} \\
& \leq \Theta\left(\frac{\gamma-\alpha}{q_{0}(\beta-\alpha)}\right)^{(\gamma-\alpha) /(\beta-\gamma)} \frac{(\beta-\gamma)}{\beta-\alpha} \\
& \quad\left(\sum_{n=a}^{b-1}|r(n)|^{\alpha /(\alpha-1)}\right)^{(\alpha-1)(\beta-\alpha) /[\alpha(\beta-\gamma)]} \sum_{n=a}^{b-1}\left|\Delta^{m} u(n)\right|^{\alpha}
\end{aligned}
$$

where $\Theta$ is defined as in (12). From (28) and (29), we have

$$
\begin{aligned}
\sum_{n=a}^{b-1} \mid & \left.\Delta^{m} u(n)\right|^{\alpha} \\
\leq & \Theta\left(\frac{\gamma-\alpha}{q_{0}(\beta-\alpha)}\right)^{(\gamma-\alpha) /(\beta-\gamma)} \frac{(\beta-\gamma)}{\beta-\alpha} \\
& \cdot\left(\sum_{n=a}^{b-1}|r(n)|^{\alpha /(\alpha-1)}\right)^{(\alpha-1)(\beta-\alpha) /[\alpha(\beta-\gamma)]} \sum_{n=a}^{b-1}\left|\Delta^{m} u(n)\right|^{\alpha} .
\end{aligned}
$$

Now, we claim that $\sum_{n=a}^{b-1}\left|\Delta^{m} u(n)\right|^{\alpha}>0$. In fact, if the above inequality is not true, we have $\sum_{n=a}^{b-1}\left|\Delta^{m} u(n)\right|^{\alpha}=0$. From (16) and (22), we obtain $u(n)=0$ for $n \in \mathbb{Z}[a, b]$, which contradicts $u(n) \neq \equiv, n \in \mathbb{Z}[a, b]$. Thus dividing both sides of (30) by $\sum_{n=a}^{b-1}\left|\Delta^{m} u(n)\right|^{\alpha}$, we obtain (10) holds. This completes the proof of Theorem 2.1.

Next, we establish a Lyapunov-type inequality for Eq. (5) under the boundary condition (6). 
Theorem 2.2 If $u(n)$ is a nonzero solution of Eq. (5) satisfying the boundary conditions (6), then

$$
\begin{aligned}
1 \leq & \left(\frac{\gamma-\alpha}{q_{0}(\beta-\alpha)}\right)^{(\gamma-1) /(\beta-\gamma)} \frac{\beta-\gamma}{\beta-\alpha} \\
& \cdot\left(\sum_{n=a}^{b-1}|r(n)|^{\alpha /(\alpha-1)}\right)^{(\alpha-1)(\beta-1) /[\alpha(\beta-\gamma)]},
\end{aligned}
$$

where $q_{0}$ is defined as in (11) and

$$
\Upsilon=\frac{(b-a)^{(\gamma-1)(2 m-2 m \beta+\alpha-1) /[\alpha(\gamma-\beta)]}}{2^{(3 m-1)(\gamma-1)(\beta-1) /(\beta-\gamma)}} .
$$

Proof Choose $c \in \mathbb{Z}[a, b]$ such that $|u(c)|=\max _{n \in \mathbb{Z}[a, b]}|u(n)|$. Since (6), it follows from Lemma 2.2 that

$$
|u(c)| \leq \frac{(c-a)(b-c)}{b-a} \sum_{n=a}^{b-1}\left|\Delta^{2} u(n)\right| \leq \frac{b-a}{4} \sum_{n=a}^{b-1}\left|\Delta^{2} u(n)\right|
$$

and

$$
\sum_{n=a}^{b-1}\left|\Delta^{2 i} u(n)\right| \leq \frac{(b-a)^{2}}{8} \sum_{n=a}^{b-1}\left|\Delta^{2 i+2} u(n)\right|, \quad i=1,2, \ldots, m-1
$$

From (33) and (34), we obtain

$$
\begin{aligned}
|u(c)| & \leq \frac{b-a}{4} \sum_{n=a}^{b-1}\left|\Delta^{2} u(n)\right| \\
& \leq \frac{b-a}{4} \frac{(b-a)^{2}}{8} \sum_{n=a}^{b-1}\left|\Delta^{4} u(n)\right| \\
& \leq \frac{b-a}{4} \frac{(b-a)^{4}}{8^{2}} \sum_{n=a}^{b-1}\left|\Delta^{6} u(n)\right| \\
& \leq \cdots \\
& \leq \frac{b-a}{4} \frac{(b-a)^{2(m-1)}}{8^{m-1}} \sum_{n=a}^{b-1}\left|\Delta^{2 m} u(n)\right| .
\end{aligned}
$$

Multiplying (5) by $\Delta^{2 m} u(n)$, we have

$$
\left|\Delta^{2 m} u(n)\right|^{\alpha}=r(n)|u(n)|^{\gamma-2} u(n) \Delta^{2 m} u(n)-q(n)\left|\Delta^{2 m} u(n)\right|^{\beta}, \quad n \in \mathbb{Z}[a, b] .
$$

Then we get

$$
\begin{aligned}
\left|\Delta^{2 m} u(n)\right|^{\alpha} & =r(n)|u(n)|^{\gamma-2} u(n) \Delta^{2 m} u(n)-q(n)\left|\Delta^{2 m} u(n)\right|^{\beta} \\
& \leq|r(n)||u(n)|^{\gamma-1}\left|\Delta^{2 m} u(n)\right|-q(n)\left|\Delta^{2 m} u(n)\right|^{\beta} .
\end{aligned}
$$


Lin Journal of Inequalities and Applications

(2020) 2020:80

Page 8 of 12

Summing (37) from $a$ to $b-1$, we have

$$
\sum_{n=a}^{b-1}\left|\Delta^{2 m} u(n)\right|^{\alpha} \leq \sum_{n=a}^{b-1}|r(n)||u(n)|^{\gamma-1}\left|\Delta^{2 m} u(n)\right|-\sum_{n=a}^{b-1} q(n)\left|\Delta^{2 m} u(n)\right|^{\beta} .
$$

For the first summation on the right-hand side of (38), from (35) we obtain

$$
\begin{aligned}
& \sum_{n=a}^{b-1}|r(n)||u(n)|^{\gamma-1}\left|\Delta^{2 m} u(n)\right| \\
& \quad \leq\left(\frac{b-a}{4}\right)^{\gamma-1} \frac{(b-a)^{2(m-1)(\gamma-1)}}{8^{(m-1)(\gamma-1)}}\left(\sum_{n=a}^{b-1}\left|\Delta^{2 m} u(n)\right|\right)^{\gamma-1} \sum_{n=a}^{b-1}|r(n)|\left|\Delta^{2 m} u(n)\right| .
\end{aligned}
$$

On the other hand, from the discrete Hölder inequality (21) with $f(n)=1, g(n)=\left|\Delta^{2 m} u(n)\right|$, $\rho=\alpha /(\alpha-1)$ and $\nu=\alpha$, we have

$$
\begin{aligned}
\sum_{n=a}^{b-1}\left|\Delta^{2 m} u(n)\right| & \leq\left(\sum_{n=a}^{b-1} 1^{\alpha /(\alpha-1)}\right)^{(\alpha-1) / \alpha}\left(\sum_{n=a}^{b-1}\left|\Delta^{2 m} u(n)\right|^{\alpha}\right)^{1 / \alpha} \\
& =(b-a)^{(\alpha-1) / \alpha}\left(\sum_{n=a}^{b-1}\left|\Delta^{2 m} u(n)\right|^{\alpha}\right)^{1 / \alpha}
\end{aligned}
$$

and with $f(n)=|r(n)|, g(n)=\left|\Delta^{2 m} u(n)\right|, \rho=\alpha /(\alpha-1)$ and $\nu=\alpha$, we get

$$
\sum_{n=a}^{b-1}|r(n)|\left|\Delta^{2 m} u(n)\right| \leq\left(\sum_{n=a}^{b-1}|r(n)|^{\alpha /(\alpha-1)}\right)^{(\alpha-1) / \alpha}\left(\sum_{n=a}^{b-1}\left|\Delta^{2 m} u(n)\right|^{\alpha}\right)^{1 / \alpha}
$$

From (39)-(41), we have

$$
\begin{aligned}
& \sum_{n=a}^{b-1}|r(n)||u(n)|^{\gamma-1}\left|\Delta^{2 m} u(n)\right| \\
& \leq \frac{(b-a)^{(\gamma-1)[2 m-1+(\alpha-1) / \alpha]}}{4^{\gamma-1} 8^{(m-1)(\gamma-1)}}\left(\sum_{n=a}^{b-1}\left|\Delta^{2 m} u(n)\right|^{\alpha}\right)^{(\gamma-1) / \alpha} \\
& \quad \cdot\left(\sum_{n=a}^{b-1}|r(n)|^{\alpha /(\alpha-1)}\right)^{(\alpha-1) / \alpha}\left(\sum_{n=a}^{b-1}\left|\Delta^{2 m} u(n)\right|^{\alpha}\right)^{1 / \alpha} \\
& =\frac{(b-a)^{(\gamma-1)[2 m-1+(\alpha-1) / \alpha]}}{4^{\gamma-1} 8^{(m-1)(\gamma-1)}}\left(\sum_{n=a}^{b-1}|r(n)|^{\alpha /(\alpha-1)}\right)^{(\alpha-1) / \alpha}\left(\sum_{n=a}^{b-1}\left|\Delta^{2 m} u(n)\right|^{\alpha}\right)^{\gamma / \alpha} .
\end{aligned}
$$

By (38) and (42), we get

$$
\begin{aligned}
& \sum_{n=a}^{b-1}\left|\Delta^{2 m} u(n)\right|^{\alpha} \\
& \quad \leq \frac{(b-a)^{(\gamma-1)[2 m-1+(\alpha-1) / \alpha]}}{4^{\gamma-1} 8^{(m-1)(\gamma-1)}}\left(\sum_{n=a}^{b-1}|r(n)|^{\alpha /(\alpha-1)}\right)^{(\alpha-1) / \alpha}\left(\sum_{n=a}^{b-1}\left|\Delta^{2 m} u(n)\right|^{\alpha}\right)^{\gamma / \alpha}
\end{aligned}
$$




$$
\begin{aligned}
& -\sum_{n=a}^{b-1} q(n)\left|\Delta^{2 m} u(n)\right|^{\beta} \\
\leq & \frac{(b-a)^{(\gamma-1)[2 m-1+(\alpha-1) / \alpha]}}{4^{\gamma-1} 8^{(m-1)(\gamma-1)}}\left(\sum_{n=a}^{b-1}|r(n)|^{\alpha /(\alpha-1)}\right)^{(\alpha-1) / \alpha}\left(\sum_{n=a}^{b-1}\left|\Delta^{2 m} u(n)\right|^{\alpha}\right)^{\gamma / \alpha} \\
& -q_{0} \sum_{n=a}^{b-1}\left|\Delta^{2 m} u(n)\right|^{\beta}
\end{aligned}
$$

where $q_{0}$ is defined as in (11). On the other hand, by using Hölder inequality (21) with $f(n)=1, g(n)=\left|\Delta^{2 m} u(n)\right|, \rho=\beta /(\beta-\alpha)$ and $v=\beta / \alpha$, we obtain

$$
\begin{aligned}
\sum_{n=a}^{b-1}\left|\Delta^{2 m} u(n)\right|^{\alpha} & \leq\left(\sum_{n=a}^{b-1} 1^{\beta /(\beta-\alpha)}\right)^{(\beta-\alpha) / \beta}\left(\sum_{n=a}^{b-1}\left(\left|\Delta^{2 m} u(n)\right|^{\alpha}\right)^{\beta / \alpha}\right)^{\alpha / \beta} \\
& =(b-a)^{(\beta-\alpha) / \beta}\left(\sum_{n=a}^{b-1}\left|\Delta^{2 m} u(n)\right|^{\beta}\right)^{\alpha / \beta}
\end{aligned}
$$

Therefore,

$$
(b-a)^{(\alpha-\beta) / \alpha}\left(\sum_{n=a}^{b-1}\left|\Delta^{2 m} u(n)\right|^{\alpha}\right)^{\beta / \alpha} \leq \sum_{n=a}^{b-1}\left|\Delta^{2 m} u(n)\right|^{\beta} .
$$

From (43) and (45), we get

$$
\begin{aligned}
& \sum_{n=a}^{b-1}\left|\Delta^{2 m} u(n)\right|^{\alpha} \\
& \leq \frac{(b-a)^{(\gamma-1)[2 m-1+(\alpha-1) / \alpha]}}{4^{\gamma-1} 8^{(m-1)(\gamma-1)}}\left(\sum_{n=a}^{b-1}|r(n)|^{\alpha /(\alpha-1)}\right)^{(\alpha-1) / \alpha}\left(\sum_{n=a}^{b-1}\left|\Delta^{2 m} u(n)\right|^{\alpha}\right)^{\gamma / \alpha} \\
& \quad-(b-a)^{(\alpha-\beta) / \alpha} q_{0}\left(\sum_{n=a}^{b-1}\left|\Delta^{2 m} u(n)\right|^{\alpha}\right)^{\beta / \alpha} .
\end{aligned}
$$

For the right-hand of (46), from the inequality (7) in Lemma 2.1 with

$$
M=\frac{(b-a)^{(\gamma-1)[2 m-1+(\alpha-1) / \alpha]}}{4^{\gamma-1} 8^{(m-1)(\gamma-1)}}\left(\sum_{n=a}^{b-1}|r(n)|^{\alpha /(\alpha-1)}\right)^{(\alpha-1) / \alpha},
$$

$x=\sum_{n=a}^{b-1}\left|\Delta^{2 m} u(n)\right|^{\alpha}, N=(b-a)^{(\alpha-\beta) / \alpha} q_{0}, \lambda=\gamma / \alpha$, and $\theta=\beta / \alpha$, we have

$$
\begin{aligned}
& \frac{(b-a)^{(\gamma-1)[2 m-1+(\alpha-1) / \alpha]}}{4^{\gamma-1} 8^{(m-1)(\gamma-1)}}\left(\sum_{n=a}^{b-1}|r(n)|^{\alpha /(\alpha-1)}\right)^{(\alpha-1) / \alpha}\left(\sum_{n=a}^{b-1}\left|\Delta^{2 m} u(n)\right|^{\alpha}\right)^{\gamma / \alpha} \\
& -(b-a)^{(\alpha-\beta) / \alpha} q_{0}\left(\sum_{n=a}^{b-1}\left|\Delta^{2 m} u(n)\right|^{\alpha}\right)^{\beta / \alpha}
\end{aligned}
$$




$$
\begin{aligned}
\leq & \Upsilon\left(\frac{\gamma-\alpha}{q_{0}(\beta-\alpha)}\right)^{(\gamma-1) /(\beta-\gamma)} \frac{\beta-\gamma}{\beta-\alpha} \\
& \cdot\left(\sum_{n=a}^{b-1}|r(n)|^{\alpha /(\alpha-1)}\right)^{(\alpha-1)(\beta-1) /[\alpha(\beta-\gamma)]} \sum_{n=a}^{b-1}\left|\Delta^{2 m} u(n)\right|^{\alpha},
\end{aligned}
$$

where $\Upsilon$ is defined as in (32). From (46) and (47), we have

$$
\begin{aligned}
\sum_{n=a}^{b-1} \mid & \left.\Delta^{2 m} u(n)\right|^{\alpha} \\
\leq & \Upsilon\left(\frac{\gamma-\alpha}{q_{0}(\beta-\alpha)}\right)^{(\gamma-1) /(\beta-\gamma)} \frac{\beta-\gamma}{\beta-\alpha} \\
& \cdot\left(\sum_{n=a}^{b-1}|r(n)|^{\alpha /(\alpha-1)}\right)^{(\alpha-1)(\beta-1) /[\alpha(\beta-\gamma)]} \sum_{n=a}^{b-1}\left|\Delta^{2 m} u(n)\right|^{\alpha} .
\end{aligned}
$$

Now, we claim that $\sum_{n=a}^{b-1}\left|\Delta^{2 m} u(n)\right|^{\alpha}>0$. In fact, if the above inequality is not true, we have $\sum_{n=a}^{b-1}\left|\Delta^{2 m} u(n)\right|^{\alpha}=0$, then $\left|\Delta^{2 m} u(n)\right|=0$ for $n \in \mathbb{Z}[a, b-1]$. So we get $\sum_{n=a}^{b-1}\left|\Delta^{2 m} u(n)\right|=0$. From (35), we obtain $u(c)=0$, then we have $u(n)=0$ for $n \in \mathbb{Z}[a, b]$, which contradicts $u(n) \not \equiv 0, n \in \mathbb{Z}[a, b]$. Thus dividing both sides of (48) by $\sum_{n=a}^{b-1}\left|\Delta^{2 m} u(n)\right|^{\alpha}$, we obtain (31) holds. This completes the proof of Theorem 2.2.

\section{Applications}

In this section, we present some examples and applications of our main results. First, we consider the nonexistence for solutions of the BVP consisting of Eq. (4) and the boundary conditions (2).

\section{Theorem 3.1 Assume}

$$
\Theta\left(\frac{\gamma-\alpha}{q_{0}(\beta-\alpha)}\right)^{(\gamma-\alpha) /(\beta-\gamma)} \frac{(\beta-\gamma)}{\beta-\alpha}\left(\sum_{n=a}^{b-1}|r(n)|^{\alpha /(\alpha-1)}\right)^{(\alpha-1)(\beta-\alpha) /[\alpha(\beta-\gamma)]}<1
$$

where $q_{0}$ and $\Theta$ are defined as in (11) and (12). Then BVP (4), (2) has no nontrivial solution.

Proof Assume the contrary. Then BVP (4), (2) has a nontrivial solution $u(n)$. By Theorem 2.1, inequality (10) holds. This contradicts assumption (49). This completes the proof of Theorem 3.1.

Next, we give an application of the obtained Lyapunov-type inequality for the following eigenvalue problem:

$$
\begin{aligned}
& \left|\Delta^{2 m} u(n)\right|^{\alpha-2} \Delta^{2 m} u(n)+q(n)\left|\Delta^{2 m} u(n)\right|^{\beta-2} \Delta^{2 m} u(n)-\lambda r(n)|u(n)|^{\gamma-2} u(n)=0, \\
& \quad n \in \mathbb{Z}[a, b],
\end{aligned}
$$

with the following boundary conditions:

$$
\Delta^{2 i} u(a)=\Delta^{2 i} u(b)=0, \quad i=0,1, \ldots, m-1 ; \quad u(n) \not \equiv 0, \quad n \in \mathbb{Z}[a, b],
$$


where $m \in \mathbb{N}, \lambda>0$ is a parameter, $1<\alpha<\gamma<\beta$ are constants, $r(n)$ and $q(n)$ are realvalued functions defined on $\mathbb{Z}$ with $q(n)>0$. Thus, if there exists a nontrivial solution $u(n)$ of BVP (50), (51), from Theorem 2.2, we have

$$
\begin{aligned}
\lambda \geq & \left(\sum_{n=a}^{b-1}|r(n)|^{\alpha /(\alpha-1)}\right)^{(1-\alpha) / \alpha}\left(\frac{\gamma-\alpha}{q_{0}(\beta-\alpha)}\right)^{(1-\gamma) /(\beta-1)} \\
& \cdot\left(\frac{\beta-\gamma}{\Upsilon(\beta-\alpha)}\right)^{(\beta-\gamma) /(\beta-1)}
\end{aligned}
$$

where $q_{0}$ and $\Upsilon$ are defined as in (11) and (32).

\section{Acknowledgements}

The author is indebted to the anonymous referees for their valuable suggestions and helpful comments which helped improve the paper significantly.

\section{Funding}

This research was supported by the Natural Science Foundation of Shandong Province (China) (No. ZR2018MA018), and the National Natural Science Foundation of China (No. 61873144).

\section{Competing interests}

The author declares that there is no conflict of interests regarding the publication of this paper.

\section{Authors' contributions}

$\mathrm{HL}$ organized and wrote this paper. Further, he examined all the steps of the proofs in this research. The author read and approved the final manuscript.

\section{Publisher's Note}

Springer Nature remains neutral with regard to jurisdictional claims in published maps and institutional affiliations.

Received: 23 June 2019 Accepted: 4 March 2020 Published online: 30 March 2020

\section{References}

1. Agarwal, R.P., Bohner, M., Peterson, A.: Inequalities on time scales: a survey. Math. Inequal. Appl. 4, 535-557 (2001)

2. Liu, H.D.: On some nonlinear retarded Volterra-Fredholm type integral inequalities on time scales and their applications. J. Inequal. Appl. 2018, Article ID 211 (2018)

3. Zhao, D.L., Yuan, S.L., Liu, H.D.: Stochastic dynamics of the delayed chemostat with Lévy noises. Int. J. Biomath. 12(5), Article ID 1950056 (2019)

4. Liu, H.D., Meng, F.W.: Some new generalized Volterra-Fredholm type discrete fractional sum inequalities and their applications. J. Inequal. Appl. 2016, Article ID 213 (2016)

5. Li, Y.N., Sun, Y.G., Meng, F.W.: Exponential stabilization of switched time-varying systems with delays and disturbances. Appl. Math. Comput. 324, 131-140 (2018)

6. Zou, C., Xia, Y., Pinto, M.: Hölder continuity of topological equivalence functions of DEPCAGs. Sci. China Math. (2019). https://doi.org/10.1007/s11425-018-9483-X

7. Liu, H.D.: Some new half-linear integral inequalities on time scales and applications. Discrete Dyn. Nat. Soc. 2019, Article ID $9860302(2019)$

8. Saker, S.H.: Some nonlinear dynamic inequalities on time scales. Math. Inequal. Appl. 14, 633-645 (2011)

9. Liu, H.D.: Some new integral inequalities with mixed nonlinearities for discontinuous functions. Adv. Differ. Equ. 2018, Article ID 22 (2018)

10. Xia, Y., Cheng, S.: Quasi-uniformly asymptotic stability and existence of almost periodic solutions of difference equations with applications in population dynamic systems. J. Differ. Equ. Appl. 14, 59-81 (2008)

11. Liu, H.D., Meng, F.W.: Some new nonlinear integral inequalities with weakly singular kernel and their applications to FDEs. J. Inequal. Appl. 2015, Article ID 209 (2015)

12. Li, Y.N., Sun, Y.G., Meng, F.W.: New criteria for exponential stability of switched time-varying systems with delays and nonlinear disturbances. Nonlinear Anal. Hybrid Syst. 26, 284-291 (2017)

13. Zhao, D.L., Liu, H.D.: Coexistence in a two species chemostat model with Markov switchings. Appl. Math. Lett. 94, 266-271 (2019)

14. Sun, Y.G., Tian, Y.Z., Xie, X.J.: Stabilization of positive switched linear systems and its application in consensus of multiagent systems. IEEE Trans. Autom. Control 62, 6608-6613 (2017)

15. Liu, H.D., Meng, F.W.: Existence of positive periodic solutions for a predator-prey system of Holling type IV function response with mutual interference and impulsive effects. Discrete Dyn. Nat. Soc. 2015, Article ID 138984 (2015)

16. Wang, J.F., Meng, F.W., Gu, J.: Estimates on some power nonlinear Volterra-Fredholm type dynamic integral inequalities on time scales. Adv. Differ. Equ. 2017, Article ID 257 (2017)

17. Liu, H.D., Meng, F.W., Liu, P.C.: Oscillation and asymptotic analysis on a new generalized Emden-Fowler equation. Appl. Math. Comput. 219(5), 2739-2748 (2012) 
18. Zhao, D.L., Yuan, S.L., Liu, H.D.: Random periodic solution for a stochastic SIS epidemic model with constant population size. Adv. Differ. Equ. 2018, Article ID 64 (2018)

19. Zou, C., Xia, Y., Pinto, M., Shi, J., Bai, Y.: Boundness and linearisation of a class of differential equations with piecewise constant argument. Qual. Theory Dyn. Syst. 18(2), 495-531 (2019)

20. Liu, H.D., Li, C.Y., Shen, F.C.: A class of new nonlinear dynamic integral inequalities containing integration on infinite interval on time scales. Adv. Differ. Equ. 2019. Article ID 311 (2019)

21. Sun, Y.G., Meng, F.W.: Reachable set estimation for a class of nonlinear time-varying systems. Complexity 2017, Article ID 5876371 (2017)

22. Tunç, E., Liu, H.D.: Oscillatory behavior for second-order damped differential equation with nonlinearities including Riemann-Stieltjes integrals. Electron. J. Differ. Equ. 2018, Article ID 54 (2018)

23. Liu, H.D.: A class of retarded Volterra-Fredholm type integral inequalities on time scales and their applications. J. Inequal. Appl. 2017, Article ID 293 (2017)

24. Feng, Q.H., Meng, F.W., Zheng, B.: Gronwall-Bellman type nonlinear delay integral inequalities on time scale. J. Math. Anal. Appl. 382, 772-784 (2011)

25. Liu, H.D., Meng, F.W.: Interval oscillation criteria for second-order nonlinear forced differential equations involving variable exponent. Adv. Differ. Equ. 2016, Article ID 291 (2016)

26. Tian, Y.Z., Fan, M., Meng, F.W.: A generalization of retarded integral inequalities in two independent variables and their applications. Appl. Math. Comput. 221, 239-248 (2013)

27. Liu, H.D., Meng, F.W.: Nonlinear retarded integral inequalities on time scales and their applications. J. Math. Inequal. 12(1), 219-234 (2018)

28. Lyapunov, A.M.: Probleme général de la stabilité du mouvement. Ann. Fac. Sci. Univ. Toulouse 2, 27-247 (1907) (French translation of a Russian paper dated 1893). Reprinted as Ann. Math. Stud., vol. 17, Princeton University Press (1947)

29. Agarwal, R.P., Özbekler, A.: Disconjugacy via Lyapunov and Vallée-Poussin type inequalities for forced differential equations. Appl. Math. Comput. 265, 456-468 (2015)

30. Liu, H.D.: Lyapunov-type inequalities for certain higher-order half-linear differential equations. J. Math. Inequal. 13(4), 1159-1170 (2019)

31. Cheng, S.S.: Lyapunov inequalities for differential and difference equations. Fasc. Math. 23, 25-41 (1991)

32. Dhar, S., Kong, Q.K.: Lyapunov-type inequalities for $\alpha$-th order fractional differential equations with $2<\alpha \leq 3$ and fractional boundary conditions. Electron. J. Differ. Equ. 2017, Article ID 203 (2017)

33. Eliason, S.B.: Lyapunov inequalities and bounds on solutions of certain second order equations. Can. Math. Bull. 17(4) 499-504 (1974)

34. Guseinov, G.S., Kaymakcalan, B.: Lyapunov inequalities for discrete linear Hamiltonian systems. Comput. Math. Appl. 45, 1399-1416 (2003)

35. Unal, M., Çakmak, D., Tiryaki, A.: A discrete analogue of Lyapunov-type inequalities for nonlinear systems. Comput. Math. Appl. 55, 2631-2642 (2008)

36. Liu, H.D.: Lyapunov-type inequalities for second-order boundary value problems with a parameter. Discrete Dyn. Nat. Soc. 2020, Article ID $1209260(2020)$

37. Liu, X.G., Tang, M.L.: Lyapunov-type inequality for higher order difference equations. Appl. Math. Comput. 232 666-669 (2014)

38. Tang, X.H., Zhang, M.: Lyapunov inequalities and stability for linear Hamiltonian systems. J. Differ. Equ. 252, 358-381 (2012)

39. Zhang, Q.M., Tang, X.H.: Lyapunov-type inequalities for even order difference equations. Appl. Math. Lett. 25, 1830-1834 (2012)

40. Tiryaki, A., Çakmak, D., Aktas, M.F.: Lyapunov-type inequalities for a certain class of nonlinear systems. Comput. Math. Appl. 64, 1804-1811 (2012)

41. Yang, X.J., Lo, K.M.: Lyapunov-type inequalities for a class of higher-order linear differential equations with anti-periodic boundary conditions. Appl. Math. Lett. 34, 33-36 (2014)

42. Zhang, Q.M., Tang, X.H.: Lyapunov inequalities and stability for discrete linear Hamiltonian system. Appl. Math. Comput. 218, 574-582 (2011)

43. Liu, H.D.: Lyapunov-type inequalities for certain higher-order difference equations with mixed non-linearities. Adv. Differ. Equ. 2018, Article ID 229 (2018)

44. Liu, H.D.: An improvement of the Lyapunov inequality for certain higher order differential equations. J. Inequal. Appl. 2018, Article ID 215 (2018)

45. Jeli, M., Samet, B.: On Lyapunov-type inequalities for (p,q)-Laplacian systems. J. Inequal. Appl. 2017, Article ID 100 (2017) 